\title{
Analysis of Student Preconceptions Related to Telecommunications and Qual- ity of Service
}

\section{Prof. Mark J. Indelicato, Rochester Institute of Technology (CAST)}

Mark J. Indelicato is an associate professor in the College of Applied Science and Technology (CAST) in the department of Electrical, Computer and Telecommunications Engineering Technology at the Rochester Institute of Technology since 1990. Previously, he was a Large Business Systems Communications Engineer for NEC America, specializing in large scale deployment of voice and data network switching equipment. He teaches in the Master of Science Telecommunications Engineering Technology program and conducts research in Real Time Audio Collaboration (RTAC) and the feasibility, logistics and implementation of live recording sessions carried and delivered over IP networks, Anomaly Detection for Music developing recommender systems for listeners and consumers and 3-D Audio perception. Indelicato holds a Bachelor of Engineering in Electrical Engineering (BEEE) from Manhattan College, a Master of Science in Information Systems Engineering (MSISE) from Brooklyn Polytechnic University and is an active member of IEEE, ASEE, and the Audio Engineering Society (AES).

\section{Prof. George H Zion, Rochester Institute of Technology (CAST)}

George H. Zion Professor - Computer Engineering Technology Affiliate Director - Project Lead The Way Rochester Institute of Technology

Professor Zion has been working with Project Lead the Way since 1997, first in the capacity as a University Affiliate Professor for the Digital Electronics curriculum and for the last four year as the Affiliate Director at RIT.

His teaching and research interests include software development for embedded systems design and K-12 pre-engineering activities.

Professor Zion received his B.S. degree in Electrical Engineering Technology and M.S. degree in Computer Science from Rochester Institute of Technology. Prior to joining RIT, he was an R\&D design engineer for Microwave Filter Company in Syracuse, New York. He is a member of ASEE and IEEE.

\section{Prof. Joseph Alan Nygate, Rochester Institute of Technology (CAST)}

Current position Associate Professor, College of Applied Science and Technology, RIT

Previous employment 10 years as Vice President of Technology and Architecture, Amdocs 6 years as Director of Architecture and Business Development, Nortel Networks 10 years, MTS Research and Development, AT\&T Bell Labs

Education PhD Computer Engineering, 1994, Case Western Reserve University, USA - AT\&T Bell Labs, PhD Scholar MSc Computer Science, 1985, Weizmann Institute of Science, Israel MSc Applied Mathematics, 1985, Weizmann Institute of Science, Israel BSc Computer Science and Mathematics, 1982, Ben-Gurion University, Israel

Interests Big Data Applications in Telecommunications Software Defined Networks - operations, management and orchestration Artificial Intelligence - expert systems, intelligent agents, reinforcement learning Self-Organizing Networks Number Theory 


\title{
Analysis of Student Preconceptions Related to Quality of Service in Telecommunications
}

\begin{abstract}
This study evaluates STEM students’ preconceptions regarding Quality of Service (QoS) in telecommunications and networking with the goal of understanding the nature of these preconceptions to improve student learning in this discipline. In this study we explain the importance of identifying preconceptions with which students enter our classrooms and illustrate a mechanism successfully used in this identification process. Researchers have explained it incumbent on educators to address preconceptions in order to effectively change student beliefs ${ }^{1}$. Analyzing the causes of these will allow teachers to instruct effectively from the start of the topic rather that lose time by re-teaching the material. As networks grow to handle increasing demands for capacity and QoS, telecommunications professionals are responsible for engineering and managing these networks. A solid understanding of factors that affect QoS is imperative and, as such, telecommunications networking instruction must be properly informed.
\end{abstract}

\section{Introduction}

Preconceptions influence our understanding and have a direct effect on how we process information to learn, solve problems and arrive at conclusions ${ }^{2}$. John Clement's work with students in introductory mechanics courses discusses "conceptual primitives" which manifest themselves in "stable, alternate views of force and acceleration" 3 . Clement's studies pair force and acceleration as concepts that interplay in students "understanding of motion related to objects.” Similarly, this research considered paired concepts of bandwidth and QoS, resource reservation and overall QoS and network utilization verses efficiency. The study of these paired concepts inform student understanding of IP networks and their performance. The first step in this study is to establish the existence and nature of these preconceptions. Subsequent studies will offer instructional techniques for conceptual change. For this first study formative assessment in the form of a low-stakes pre-test was used to inform instructional strategies to address misconceptions associated with concepts of bandwidth, resource reservation, network utilization and efficiency in an undergraduate level telecommunications course.

Undergraduate students enrolled in a networking technologies course as part of their telecommunications engineering technology option were asked three basic questions regarding QoS prior to instruction. Their responses were analyzed and compared to the responses to the identical questions following instruction, creating paired response data. Individual question scores, average scores and normalized gain values were generated for each student's performance for each question overall score. Results indicate that students had more incorrect preconceptions regarding bandwidth than with resource reservation, network utilization and efficiency. This study will expand the breadth of knowledge about student preconceptions in 
STEM by including the subject of QoS in the telecommunications discipline, identify the preconception(s), statistically analyze the effects of these preconceptions and offer instructional insights than can ameliorate or eliminate negative effects on student learning related to these concepts.

\section{Concepts}

The following explanations of each of the paired concepts are provided for readers not familiar with telecommunications and QoS.

In general quality of service, as it applies to IP networks, is the study and practice of providing pre-determined service level agreements (SLAs) of voice, data and multimedia services using a variety of techniques. All traffic in an IP network is considered "data". The telecommunications industry uses the term data to include traffic such as file transfers, email, web pages, etc. Voice and multi-media are data that require different service levels due to the real time nature of the delivery and content. Pre-determined levels are metrics of a service level agreement that pertain to service quality as provided by the service provider to the customer. These metrics may be degrees of latency, redundancy, guaranteed bit rate and commitment to deliver customer traffic. Although any network may employ QoS techniques, they are especially important for networks that serve large numbers of users and provide many types of services. As such, commercial carrier and private enterprise networks must employ QoS, while smaller networks such as home networks or small local area networks do not usually employ QoS.

Bandwidth in data communications networks quantifies the transmission speed, in bits per second (bps), of a communications channel or "line". These lines connect switching devices, servers and databases, etc that contain the routing, switching, security and information capabilities of IP networks ${ }^{4}$. For example, a gigabit Ethernet connection is 1,000,000,000 bits per second (bps) or 1000 megabits per second (Mbps).

Quality of Service (QoS) for networks is an industry-wide set of standards and mechanisms for ensuring high-quality performance for critical applications. By using QoS mechanisms, network administrators can use existing resources efficiently and ensure the required level of service without reactively expanding or over- provisioning their networks. The QoS concept of quality is one in which the requirements of some applications and users are more critical than others which means that some traffic needs preferential treatment ${ }^{5}$. For example, bank and financial institution currency trades are more critical than Google Mail and will receive priority service levels.

Resource Reservation Protocol (RSVP) is used to allocate network resources for pre-defined applications between a sender and a receiver in an effort to assure a specific QoS level for those applications ${ }^{6}$. 
Network Utilization is the relationship between usage levels vs capacity. For example a $1 \mathrm{Mbps}$ transmission line experiencing an average usage of $500 \mathrm{Kbps}$ is said to be 50 percent utilized ${ }^{7}$.

Network efficiency can be assessed by packet latency or the time it takes to deliver information from sender to receiver ${ }^{8}$. Networks will exhibit exponentially increasing latency, becoming less efficient, as utilization approaches 60 to 70 percent.

\section{Methodology}

The concepts are studied using three questions evaluated pre-instruction and post-instruction. The terms "PRE" and "POST" are used in this discussion. Three, two part survey questions are presented to 24 students. The first part (Part A) requires a simple "Yes" or "No" response. The second part (Part B) requires an explanation of why the "Yes" or "No" answer was chosen. The two part question is designed to inform the instructor if a student guesses or has a misunderstanding by identifying "disordered pairs". An incorrect "yes" or "no" response paired with a correct explanation indicates a lack of understanding or a "misunderstanding". This disordered pair is defined as "Scenario 1". A correct "Yes" or "No" response paired with an incorrect explanation, indicates the student guessed. This is identified as "Scenario 2". Instances of misunderstanding and guessing are identified. Each part of the question is assigned a metric or maximum point total. The scores for each part are summed and represent the total score for that question. Each question is a total of 5 points, resulting in a total of 15 points for all three questions. For each of the PRE and POST instruction surveys, the following data is collected:

- Individual question scores for each student - Parts A and B individually

- Individual question scores for each student - Sum of parts A and B

- Average student score for each question - Parts A and B individually

- Average student score for each question - Sum of parts A and B

- Total student score for all three questions - Q-1 + Q-2 + Q-3

- Average total student score for all three questions $-\mathrm{Q}-1+\mathrm{Q}-2+\mathrm{Q}-3$

- Normalized Gain for each question (Parts A and B together). Normalized Gain is the difference in the POST vs PRE score divided by the class average for that particular question.

- The difference in PRE vs POST instruction disordered pair instances - Scenario 1 and 2 together

- The difference in PRE vs POST instruction disordered pair instances - Scenario 1

- The difference in PRE vs POST instruction disordered pair instances - Scenario 2

- Two-tailed, paired sample tests were performed on student scores for Q-1, Q-2, Q-3 and TOTAL score.

The questions used in the pre-instruction and post-instruction surveys were the same and are shown below: 


\section{Question 1 - Answer YES or NO}

Increasing bandwidth leads to increased QoS. (2 Points)

Why do you think this is so? (3 Points)

\section{Question 2 - Answer YES or NO}

Reserving bandwidth leads to increased QoS for all users. (1 point)

Why do you think this is so? (4 Points)

\section{Question 3 - Answer YES or NO}

Increased utilization indicates a more efficient system. (1 Point)

Why do you think this is so? (4 Points)

\section{$\underline{\text { Results/Analysis }}$}

For Question \#1, the mean knowledge gain for the twenty-four $(\mathrm{N}=24)$ students was 0.2083 with a standard deviation of 2.121. A two-tailed paired samples t-test on the mean knowledge for Q1 was not statistically significant $(\mathrm{t}(23)=0.4812$, $\mathrm{p}=0.635)$. Thus, on average, students' knowledge associated with the concept of bandwidth did not improve significantly over the duration of the semester (Table1).

For Question \#2, the mean knowledge gain for the twenty-four $(\mathrm{N}=24)$ students was 0.8333, with a standard deviation of 1.8337. A two-tailed paired samples t-test on the mean knowledge for Q2 was statistically significant $(\mathrm{t}(23)=2.226, \mathrm{p}<0.05)$. Thus, on average, students' knowledge associated with resource reservation improved significantly over the duration of the semester (Table 1).

For Question \#3, the mean knowledge gain for the twenty-four $(\mathrm{N}=24)$ students was 2.3750, with a standard deviation of 2.7790. A two-tailed paired samples t-test on the mean knowledge for Q3 was statistically significant $(\mathrm{t}(23)=4.187$, $\mathrm{p}<0.05)$. Thus, on average, students' knowledge associated with network utilization improved significantly over the duration of the semester (Table1). 


\begin{tabular}{|c|c|c|c|c|c|c|c|c|c|}
\hline \multicolumn{10}{|c|}{ Paired Samples Test } \\
\hline & & \multicolumn{5}{|c|}{ Paired Differences } & \multirow[b]{3}{*}{$t$} & \multirow[b]{3}{*}{ df } & \multirow[b]{3}{*}{ Sig. (2-tailed) } \\
\hline & & \multirow[b]{2}{*}{ Mean } & \multirow[b]{2}{*}{ Std. Deviation } & Std. Error & \multicolumn{2}{|c|}{$\begin{array}{l}\text { 95\% Confidence Interval of the } \\
\text { Difference }\end{array}$} & & & \\
\hline & & & & Mean & Lower & Upper & & & \\
\hline Pair 1 & Post_Q1T-Pre_Q1T & .2083 & 2.1209 & .4329 & -.6872 & 1.1039 & .481 & 23 & .635 \\
\hline Pair 2 & Post_Q2T - Pre_Q2T & .8333 & 1.8337 & .3743 & .0590 & 1.6076 & 2.226 & 23 & .036 \\
\hline Pair 3 & Post_Q3T - Pre_Q3T & 2.3750 & 2.7790 & .5673 & 1.2015 & 3.5485 & 4.187 & 23 & .000 \\
\hline Pair 4 & $\begin{array}{l}\text { Post_TOTAL- } \\
\text { Pre_TOTAL }\end{array}$ & 3.4167 & 4.0611 & .8290 & 1.7018 & 5.1315 & 4.122 & 23 & .000 \\
\hline
\end{tabular}

Table 1 - Paired Sample T-test

With regard to the scenarios described in the methodology section, it was noted that number of instances of misunderstanding (Scenario 1) for ALL questions decreased from 7 to 5 . The number of instances guessing (Scenario 2) for ALL questions decreased from 14 to 5 and within these instances, question 2 showed the greatest reduction in guessing (Scenario 2) from 7 to 0 instances. Question 2 showed the greatest reduction in instances of misunderstanding (Scenario 1) from 5 to 3. Question 2 also showed the greatest instance reduction of BOTH scenarios combined from 12 to 3. It is interesting to note that Question 3, though it exhibited a lower Sig. (2-tailed) value than Question 2, showed neither differences in the number of Scenario 1 nor 2 instances. It did however show the greatest Normalized Gain with a score of 1.128 (Table 2) and the greatest mean knowledge gain with a value of 2.3750 (Table 1).

$\begin{array}{rrrrr}\text { Student } & & & & \\ \mathbf{1} & -1.29032 & 0 & 2.474227 & 0.359281 \\ \mathbf{2} & 1.548387 & 1.333333 & 2.474227 & 1.724551 \\ \mathbf{3} & -1.03226 & 0 & 2.474227 & 0.431138 \\ \mathbf{4} & 2.064516 & 0 & 2.474227 & 1.293413 \\ \mathbf{5} & 0 & 0 & 2.474227 & 0.718563 \\ \mathbf{6} & 0 & 1 & 0.989691 & 0.718563 \\ \mathbf{7} & 1.548387 & -1 & 2.474227 & 0.718563 \\ \mathbf{8} & 0 & 0 & 1.979381 & 0.57485 \\ \mathbf{9} & 0 & 0 & 2.474227 & 0.718563 \\ \mathbf{1 0} & 0 & 0 & 0 & 0 \\ \mathbf{1 1} & -0.51613 & 0.333333 & 0.494845 & 0.143713 \\ \mathbf{1 2} & -0.51613 & 1 & 1.979381 & 0.862275 \\ \mathbf{1 3} & 0.516129 & -0.66667 & 0 & -0.14371 \\ \mathbf{1 4} & 1.548387 & 1.333333 & 0 & 1.005988 \\ \mathbf{1 5} & 1.032258 & 0 & 0 & 0.287425 \\ \mathbf{1 6} & 0 & 1 & 0.989691 & 0.718563 \\ \mathbf{1 7} & -2.32258 & 0 & -2.47423 & -1.36527 \\ \mathbf{1 8} & 0.516129 & 0 & 2.474227 & 0.862275 \\ \mathbf{1 9} & 0.516129 & 0 & 0.494845 & 0.287425 \\ \mathbf{2 0} & 1.032258 & 0 & 0 & 0.287425\end{array}$




$\begin{array}{lrrrr}\mathbf{2 1} & 1.032258 & 0 & 0 & 0.287425 \\ \mathbf{2 2} & -1.03226 & 0.666667 & 2.474227 & 0.718563 \\ \mathbf{2 3} & -0.51613 & 0.333333 & 1.979381 & 0.57485 \\ \mathbf{2 4} & -1.54839 & 0 & 0.494845 & -0.28743 \\ \mathbf{2 5} & 0 & 1.333333 & -0.98969 & 0.287425\end{array}$

\section{Average \\ $\begin{array}{lllll}\text { NormGain } & 0.103226 & 0.266667 & 1.128247 & 0.471377\end{array}$}

Table 2 - Normalized Gain (NG) per student per question and average NG per question

\section{$\underline{\text { Discussion }}$}

With regard to question 1, students' knowledge associated with the concept of bandwidth did not improve significantly over the duration of the semester (Table1). This may be attributed to the multi-faceted nature of the concepts inherent in this question. Students surveyed believed that increasing bandwidth "improves network performance", which is perceived as "reduced latency and response times". However, bandwidth is only one element of a robust network. Students need to understand that capacity of a network is related not only to the bandwidth of transmission lines but also to processing speeds of the switches, routers, servers, and memory dedicated within these devices. Students who don't consider nor understand these factors will mistakenly think that simply increasing bandwidth will improve QoS. These other elements related to network capacity need to be stressed and presented with their interdependencies related to QoS.

Part A of each question requires a "binary" response. A correct Yes or No response is assigned 1 or 0 points respectively. Part A of question 1 is different. The response to part A of question 1 is a Yes or No answer but the number of points assigned is 2. As it turned out, all 24 respondents received either " 0 " or " 2 " points making it clear to the researchers that a degree of latitude for this question was not necessary and 1 point should be assigned to this question.

Question 3 showed the highest normalized gain while also showing the lowest number of scenario 1 and 2 instances and change in the number of these instances. Based on the PRE Part $B$ responses, it is clear that a large number of students had little or no understanding of the paired concepts of network utilization and efficiency prior to instruction. This is evidenced by the low PRE scores and very high POST scores. These indicate the instruction was effective. Sixteen students PRE scored ZERO points for both Parts A and B. Only 5 students POST scored ZERO points for parts A and B. As such, there were very few Scenario 1 and 2 instances. A third scenario can be included. Scenario 3 would describe a 0/0 PRE score with 1/4 POST score instances. If this analysis is included, question 3 would not only show high statistical significance, high normalized gain, but also a dramatic reduction in what we can now identify as instances of "no understanding" or Scenario 3. In this study, these instances were reduced from 16 to 5 . 
The instruction was effective in making clear that in an IP network where traffic load is not deterministic and predictable, network congestion increases exponentially as utilization reaches 70\%. Network congestion results in decreased QoS levels. In fact, a network operator or service provider will attempt to maintain utilization at approximately $50 \%$ or less. Traffic shaping is a technique used to "smooth out” utilization, reducing spikes in utilization numbers for long periods of time. These concepts were stressed in the instruction phase and this effort resulted in a positive effect on student learning during the semester and dramatic increase in the number of 1/4 POST scores for this question.

Question 2 showed the best combination of Normalized Gain and decrease in Scenario 1 and 2 instances. This is most likely due to the concept being tested. It seemed reasonable to students that when resources are reserved for one population, they are not available to another.

\section{Future Work}

Graduate telecommunications students will be surveyed using the same instrument to determine if this population responds differently to the survey and instruction. Usually, these students have more networking experience than undergraduates. These graduate students are also from outside the United States of America. In addition to networking experience, culture and language may also play a significant role in the nature of these students' preconceptions, interpretation of the questions and how these affect the response data. This same survey will be given to another group of undergraduate and graduate students in the next academic year increasing the sample size $(\mathrm{N})$ and validate the survey, the data analysis process and findings. The researchers expect to increase the sample size to 150, providing a more robust data set. In addition to the numeric data, a catalog of key words used in students' PRE and POST responses will be compiled and analyzed for key word correlation to instances of misunderstanding, guessing and no knowledge of the paired concepts. The key words will be correlated to the culture and native language of the students. These key words will be analyzed to see how particular students re-phrased their responses POST instruction. A third scenario (Scenario 3 - no knowledge) will be added to the instances studied. The Rasch Item Response Theory (IRT) model will be utilized to analyze the effectiveness and validity of survey questions ${ }^{9,10}$.

\section{References}

1. Bransford, John D. , Brown, Ann L. , Cocking, Rodney R. , eds. 2000. How People Learn - Brain, Mind, Experience, and School. National Academy Press, Washington DC 
2. Cennamo, Katherine S. 1993. "Preconceptions of Mediated Instruction for Various Domains of Learning Outcomes.” International Journal of Instructional Media 20(3): 195-206.

3. Clement, John. 1982. "Students' Preconceptions in Introductory Mechanics." American Journal of Physics 50 (1): 66-71. doi: 10.1119/1.12989

4. Freeman, Roger L. 1998 "Bits, symbols, bauds, and bandwidth." Communications Magazine, IEEE 36, no. 4: 96-99. doi: 10.1109/35.667424

5. Microsoft TechNet. 2003. “What is QoS?” Accessed March 28, 2003. https://technet.microsoft.com/enus/library/cc757120(v=ws.10).aspx

6. Kim, Min-Sun, et al. 2001. “A Resource Reservation Protocol in Wireless Mobile Networks.” In Parallel Processing Workshops, 2001. International Conference on, Valencia, September 3-7. pp. 429-434.

doi:10.1109/ICPPW.2001.951982

7. Hassidim, Avinatan, et al. 2013. "Network Utilization: the Flow View,” In INFOCOM, 2013 Proceedings IEEE, Turin, April 14-17. pp.1429-1437. doi:10.1109/INFCOM.2013.6566937

8. Schneidewind, Norman F. 2012. "Network Standards.” In Computer, Network, Software, and Hardware Engineering with Applications, 211-27. John Wiley \& Sons, Inc.

9. http://www.rasch-analysis.com/rasch-measurement.htm, Copyright www.rasch-analysis.com 1990-2016.

10. Granger, C. "Rasch analysis is important to understand and use for measurement." Rasch Meas Trans 21 (2008): 1122-1123. http://www.rasch.org/rmt/rmt213d.htm 